\title{
Analysis of boundary layer in MHD flow of non-Newtonian visco-elastic fluid on stretching sheet
}

\author{
Mohammad Reza HEYDARI ${ }^{1}$, Arash Shams TALEGHANI ${ }^{2 *}$ \\ 1- Department of Mechanical Engineering, Brojerd Branch, Islamic Azad university, Brojerd, Iran \\ 2- Aerospace Research Institute, Ministry of Science, Research and Technology, Tehran, Iran
}

\begin{abstract}
This article aims to the study of fluid flow and heat transfer in non-Newtonian viscoelastic fluid magnetic hydrodynamics steady laminar flow is expanded on a horizontal plane. Among the many processes in chemical engineering, metallurgy, polymer extrusion process involves cooling the molten liquid is drawn into the cooling system, the production of paper and glass. In this process, the rate of cooling and shrinking influences very much on the final quality of the product. Equations for fluid flow and heat transfer analysis solutions are helping homotopy analysis. In this regard and in order to validate the results of the homotopy analysis, we are comparing the results of this study and other researchers published the results will be accepted. Also, the influence of various parameters on curves, speed and temperature have been investigated.
\end{abstract}

Keywords: laminar flow-border, non-Newtonian fluid, viscoelastic, spreadsheet, magnetic hydrodynamic flow

\section{Introduction}

Boundary- layer flow on continuous moving is an important type of flow that can be used in a large number of engineering processes. Aerodynamic extrusion of the plastic plates, cooling plates in a cooling bath (in the form of an electrolyte), crystal growth, metallurgy, layer-border along the liquid film is compressed in the process of distillation and polymer plate continuously, the practical applications of mobile plate as well as materials in casting processes and materials that undergo heat treatment by which the feed roller (feed roller) or on conveyor belts moving have animated feature continuous [1].

Chen \& Char [2] examined the spreadsheet linear heat transfer in the laminar boundary layers in the presence of suction / injection and the results were presented as Kummer's functions. Two different boundary conditions are: a) Prescribed surface temperature (PST), and b Prescribed heat flux (PHF). In some special circumstances Closed-form solution were provided.

\footnotetext{
*Electronic address: Taleghani@ari.ac.ir
} 
Despite the importance of non-Newtonian fluids, unfortunately, we have to admit that access to a comprehensive theory on the category of fluids (at least in the near future) is unthinkable. The main problem in this regard, is a variety of basic equations of non-Newtonian fluids [3]. In addition, the viscosity of the fluid is usually not fixed and varies with the rate of cut, which along with the elastic properties of the fluids, doubles its complexity of the topic. Fortunately, in many cases, non-Newtonian fluids exhibit viscous behavior can be well expressed by an exponential equation. The fluids are called the inclusion fluids. The most important feature is that the viscosity of fluids is not constant and is a function of shear rate. Due to the simplicity of basic equation as fluid than other types of boundary-layer of non-Newtonian fluids, it is no surprise that the initial studies of non-Newtonian fluids are specific to this particular type of fluid. $[4,5]$. In fact, we should acknowledge that even today the vast majority of scientific articles about nonNewtonian fluid is devoted to the study of this particular type of fluid [6]. However it should be noted that a fluid without the elastic properties of the fluid cannot therefore be expected to be an important flow behavior of polymer solutions and melts (which are clearly has elastic properties) even qualitatively.

Gorla \& Voss [7] discussed for the first time, the permanent and transient heat transfer fluid stagnation point in the boundary layer-two times in double dimension. The results are obtained for both permanent and non-permanent temperature behavior page transient response and detail the transient temperature step changes to the range of 5 to 1000 at page Prandtl number.

Nandeppanavar et al. [8] studied the magnetic hydrodynamic flow of viscoelastic fluid wide screen with respect to the radiation source / heat sink (non-uniform) of the energy equation. They noted that the PHF boundary conditions are more suitable for the effective cooling spreadsheet. As well as to regulate the temperature of the system should at least be considered parameters of radiation as much as possible.

In a study of Abel and Mahesha [9] made it clear that the viscoelastic fluid have the least amount of viscous waste, the best conditions are obtained for proper cooling is achieved spreadsheets. Also, it is more suitable due to the influence of thermal conductivity on temperature, the lowest thermal conductivity of the fluid for cooling operation.

The effect of fluid viscosity and thermal conductivity ranging capabilities in the viscoelastic MHD fluid on a spreadsheet was studied by Prasad and colleagues [10]. They actually expanded the work of Siddappa and Abel [11] with regard to variable fluid properties related to temperature. The aim of the study was to investigate the effects of thermal radiation parameters in the flow Walters.

\section{Description of the problem and governing equations}

Two-dimensional steady laminar flow of a viscoelastic fluid with electrical conductivity on a page spread is considered in the presence of uniform magnetic field perpendicular to the screen. 
Powered by two different -oriented and with equal force are applied and expanded along with $x$ axes (the center is kept constant). Speed of the sheet is assumed $\operatorname{asu}_{w}(x)=b x$. Uniform magnetic field is applied to the sheet along withy. The continuity equation, momentum and energy, respectively are provided for equations (1) to (3) with the help of approximation of the boundary layers. In this case, the screen rate spreads by a fixed speed of $b$. Physical geometry and coordinate system is shown in Figure 1. The problem for viscoelastic fluid is used from both fluid quadratic model (also called the fluid Walters) and the second fluid.

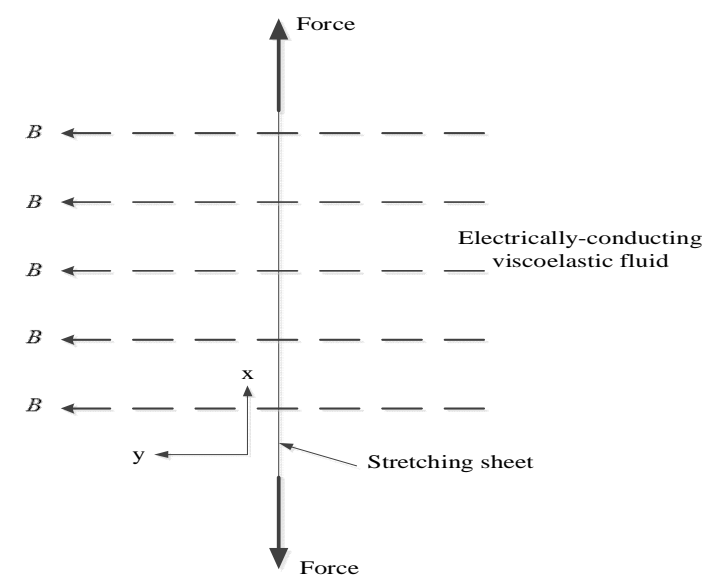

Fig. 1: The current configuration on page spread

$$
\begin{aligned}
& \frac{\partial u}{\partial x}+\frac{\partial v}{\partial y}=0 \\
& u \frac{\partial u}{\partial x}+v \frac{\partial u}{\partial y}=v \frac{\partial^{2} u}{\partial y^{2}}-\frac{\sigma B_{0}^{2} u}{\rho}- \\
& k_{0}\left(\begin{array}{l}
u \frac{\partial^{3} u}{\partial x \partial y^{2}}+v \frac{\partial^{3} u}{\partial y^{3}}+ \\
\frac{\partial u}{\partial x} \frac{\partial^{2} u}{\partial y^{2}}-\frac{\partial u}{\partial y} \frac{\partial^{2} u}{\partial x \partial y}
\end{array}\right), \\
& u \frac{\partial T}{\partial x}+v \frac{\partial T}{\partial y}=\frac{k}{\rho c_{p}} \frac{\partial^{2} T}{\partial y^{2}},
\end{aligned}
$$

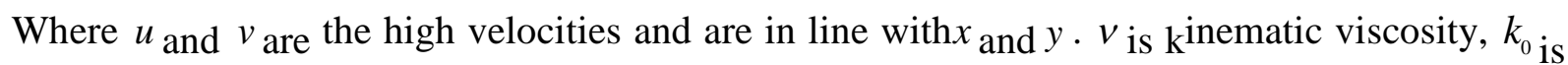
viscoelastic parameters, $k_{0}$ is viscoelastic parameter, $\sigma_{\text {is }}$ electrical distribution, $B_{0}$ is uniform magnetic field, $\rho$ fluid density, $c_{p}$ is specific heat at constant pressure, $k$ is thermal conductivity, and $T$ is temperature of the fluid. Boundary conditions for the issue of relations (4) to (6) are provided as follows:

$u=u_{w}(x), \quad v=0$, at $\quad y=0$, 
$T=T_{w}=T_{\infty}+A\left(\frac{x}{l}\right)$, at $\quad y=0$,

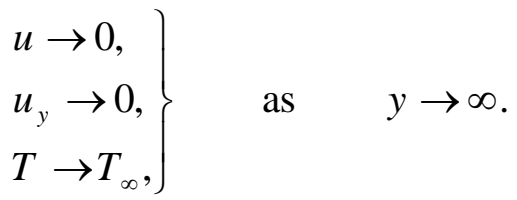

By introducing the function of flow of $\psi, u=\partial \psi / \partial y$ and $v=-\partial \psi / \partial x$ the continuity equation is satisfied by using parallel variables (7), will become momentum and energy equations for ordinary differential equations.

$\eta=\sqrt{\frac{u_{w}(x)}{v x}} y$

$\psi=\sqrt{v x u_{w}(x)} f(\eta)$

$\theta(\eta)=\frac{T-T_{\infty}}{T_{w}-T_{\infty}}$,

Where $\eta_{\text {is }}$ the thickness of the layer-border, and $f(\eta)_{\text {is }}$ dimensionless stream function. Using the above equation Placement-boundary layer equations, nonlinear differential equations are obtained.

$$
\begin{aligned}
& f^{\prime 2}(\eta)-f(\eta) f^{\prime \prime}(\eta)-f^{\prime \prime \prime}(\eta) \\
& +k_{1}\left\{\begin{array}{c}
2 f^{\prime}(\eta) f^{\prime \prime \prime}(\eta)- \\
f(\eta) f^{(4)}(\eta)-f^{\prime \prime 2}(\eta)
\end{array}\right\} \\
& +M f^{\prime}(\eta)=0, \\
& \theta^{\prime \prime}(\eta)+\operatorname{Pr}\left(\begin{array}{c}
f(\eta) \theta^{\prime}(\eta)- \\
f^{\prime}(\eta) \theta(\eta)
\end{array}\right)=0,
\end{aligned}
$$

In the above equations $k_{1}=k_{0} b / v$ is viscoelastic parameters, $M=\sigma B_{0}^{2} / b \rho_{\text {is }}$ magnetic field parameter and $\operatorname{Pr}=\mu c_{p} / k_{\text {is }}$ Prandtl number. Dimensionless boundary conditions in relationships are given below:

$$
\begin{aligned}
& f^{\prime}(\eta)=1, \quad f(\eta)=0, \quad \text { at } \quad \eta=0, \\
& f^{\prime}(\eta) \rightarrow 0, f^{\prime \prime}(\eta) \rightarrow 0, \text { as } \eta \rightarrow \infty, \\
& \theta(\eta)=1, \quad \text { at } \eta=0, \\
& \theta(\eta) \rightarrow 0, \quad \text { as } \eta \rightarrow \infty,
\end{aligned}
$$




\section{Homotopy analysis method}

The initial approximation chosen so that the boundary conditions are satisfied (in order to study in more detail the homotopy analysis method to references $[12,13]$ below):

$f_{0}(\eta)=1-e^{-\eta}$

$\theta_{0}(\eta)=e^{-\eta}$,

In the next step, linear operators should be selected for each of the equations. It is better these linear operators to be chosen so that approximations will be satisfied.

$$
\begin{aligned}
& \mathrm{L}_{f}(f)=\frac{\partial^{4} f}{\partial \eta^{4}}+\frac{\partial^{3} f}{\partial \eta^{3}}, \\
& \mathrm{~L}_{\theta}(\theta)=\frac{\partial^{2} \theta}{\partial \eta^{2}}+\frac{\partial \theta}{\partial \eta}
\end{aligned}
$$

Non-linear operators are defined to form relationships homotopy analysis for the following relationships:

$$
\begin{aligned}
& \mathrm{N}_{f}[\hat{f}(\eta ; q)]=\left(\frac{\partial \hat{f}(\eta ; q)}{\partial \eta}\right)^{2}+M \frac{\partial \hat{f}(\eta ; q)}{\partial \eta} \\
& -\hat{f}(\eta ; q) \frac{\partial^{2} \hat{f}(\eta ; q)}{\partial \eta^{2}}-\frac{\partial^{3} \hat{f}(\eta ; q)}{\partial \eta^{3}} \\
& +k_{1}\left\{\begin{array}{c}
2 \frac{\partial \hat{f}(\eta ; q)}{\partial \eta} \frac{\partial^{3} \hat{f}(\eta ; q)}{\partial \eta^{3}}- \\
\hat{f}(\eta ; q) \frac{\partial^{4} \hat{f}(\eta ; q)}{\partial \eta^{4}}-\left(\frac{\partial^{2} \hat{f}(\eta ; q)}{\partial \eta^{2}}\right)^{2}
\end{array}\right\}, \\
& \mathrm{N}_{\theta}[\hat{f}(\eta ; q), \hat{\theta}(\eta ; q)]=\frac{\partial^{2} \hat{\theta}(\eta ; q)}{\partial \eta^{2}}+ \\
& \operatorname{Pr}\left(\begin{array}{l}
\hat{f}(\eta ; q) \frac{\partial \hat{\theta}(\eta ; q)}{\partial \eta} \\
-\frac{\partial \hat{f}(\eta ; q)}{\partial \eta} \hat{\theta}(\eta ; q)
\end{array}\right),
\end{aligned}
$$

Modified equations in zero rank are changed as follows:

$$
\begin{aligned}
& (1-q) \mathrm{L}_{f}\left[\hat{f}(\eta ; q)-f_{0}(\eta)\right]= \\
& \quad q \hbar \mathrm{H}_{f}(\eta) \mathrm{N}_{f}[\hat{f}(\eta ; q), \hat{\theta}(\eta ; q)],
\end{aligned}
$$




$$
\begin{aligned}
& (1-q) \mathrm{L}_{\theta}\left[\hat{\theta}(\eta ; q)-\theta_{0}(\eta)\right]= \\
& \quad q \hbar \mathrm{H}_{\theta}(\eta) \mathrm{N}_{\theta}[\hat{f}(\eta ; q), \hat{\theta}(\eta ; q)] .
\end{aligned}
$$

Where $\hbar_{\text {is }}$ aid non-zero parameters. And $\mathrm{H}_{f}(\eta)$ and $\mathrm{H}_{\theta}(\eta)$ functions are elected according to $e^{-\eta}$. With $m$ derivative load of the zero rank equations in proportional to $q$ and dividing it by $m$ ! in $q=0$ of the $m$ rank equations as follows:

$\mathrm{L}_{f}\left[f_{m}(\eta)-\chi_{m} f_{m-1}(\eta)\right]=\hbar \mathrm{H}_{f}(\eta) R_{f, m}(\eta)$,

$\mathrm{L}_{\theta}\left[\theta_{m}(\eta)-\chi_{m} \theta_{m-1}(\eta)\right]=\hbar \mathrm{H}_{\theta}(\eta) R_{\theta, m}(\eta)$,

In the above equation, we have:

$$
\begin{gathered}
R_{f, m}(\eta)=\sum_{n=0}^{m-1}\left(\begin{array}{l}
\frac{\partial f_{n}(\eta)}{\partial \eta} \frac{\partial f_{m-1-n}(\eta)}{\partial \eta} \\
-f_{n}(\eta) \frac{\partial^{2} f_{m-1-n}(\eta)}{\partial \eta^{2}}
\end{array}\right) \\
-\frac{\partial^{3} f_{m-1}(\eta)}{\partial \eta^{3}}+M \frac{\partial f_{m-1}(\eta)}{\partial \eta} \\
+\sum_{n=0}^{m-1} k_{1}\left(\begin{array}{l}
2 \frac{\partial f_{n}(\eta)}{\partial \eta} \frac{\partial^{3} f_{m-1-n}(\eta)}{\partial \eta^{3}} \\
-f_{n}(\eta) \frac{\partial^{4} f_{m-1-n}(\eta)}{\partial \eta^{4}} \\
-\frac{\partial^{2} f_{n}(\eta)}{\partial \eta^{2}} \frac{\partial^{2} f_{m-1-n}(\eta)}{\partial \eta^{2}}
\end{array}\right), \\
R_{\theta, m}(\eta)=\frac{\partial^{2} \theta_{m-1}(\eta)}{\partial \eta^{2}}+ \\
\operatorname{Pr}\left(\begin{array}{c}
\sum_{n=0}^{m-1} \\
f_{n}(\eta) \frac{\partial \theta_{m-1-n}(\eta)}{\partial \eta} \\
-\theta_{n}(\eta) \frac{\partial f_{m-1-n}(\eta)}{\partial \eta}
\end{array}\right),
\end{gathered}
$$

Finally, using the Taylor series, we have:

$$
\begin{aligned}
& \hat{f}(\eta ; q)=f_{0}(\eta)+\sum_{m=1}^{\infty} f_{m}(\eta) q^{m}, \\
& \hat{\theta}(\eta ; q)=\theta_{0}(\eta)+\sum_{m=1}^{\infty} \theta_{m}(\eta) q^{m},
\end{aligned}
$$


$\chi_{m}=\left\{\begin{array}{ll}0 & m \leq 1 \\ 1 & m>1\end{array}\right.$,

System of equations with the boundary conditions is solved using MATHEMATICA software. $\hbar$ values have a great effect on the convergence rate. These parameter values can be selected from the right horizontal. Figure 2 shows the curve resolution analysis of homotopy with 20 stages. Optimal values of $\hbar$ should be chosen in straight lines parallel to the horizontal axis of the area.

In order to achieve the optimal value of $\hbar$ parameter, the average residual error defined as follows:

$$
\begin{gathered}
\operatorname{Res}_{f}=\left(\frac{d f(\eta)}{d \eta}\right)^{2}-f(\eta) \frac{d^{2} f(\eta)}{d \eta^{2}} \\
-\frac{d^{3} f(\eta)}{d \eta^{3}}+M \frac{d f(\eta)}{d \eta} \\
+k_{1}\left\{\begin{array}{c}
2 \frac{d f(\eta)}{d \eta} \frac{d^{3} f(\eta)}{d \eta^{3}}- \\
f(\eta) \frac{d^{4} f(\eta)}{d \eta^{4}}-\left(\frac{d^{2} f(\eta)}{d \eta^{2}}\right)^{2}
\end{array}\right\}, \\
\operatorname{Res}_{\theta}=\frac{d^{2} \theta(\eta)}{d \eta^{2}}+ \\
\operatorname{Pr}\left(f(\eta) \frac{d \theta(\eta)}{d \eta}-\frac{d f(\eta)}{d \eta} \theta(\eta)\right),
\end{gathered}
$$

In order to achieve maximum accuracy in this article, the remaining error homotopy average results of the analysis of the 20 steps to the equation (27) and (28) are drawn in Figures 3 and 4. 
Bulletin de la Société Royale des Sciences de Liège, Vol. 85, 2016, p. 21 - 33

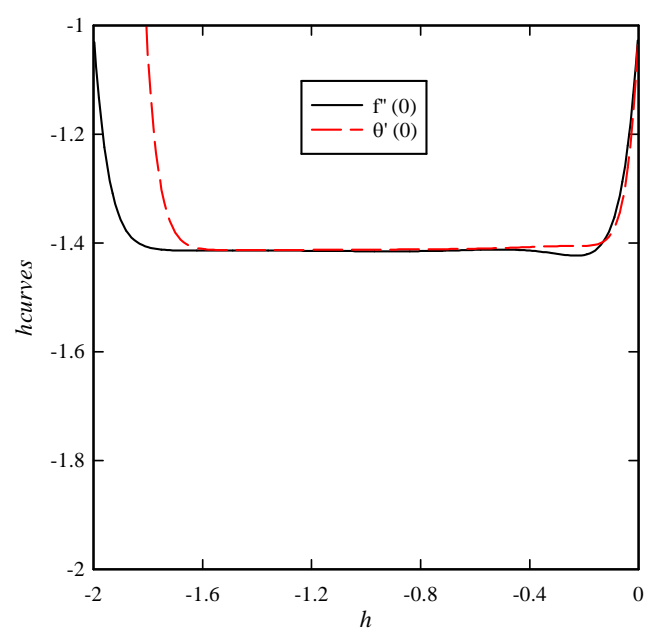

Fig. 2: Curve Twenty-stop solution for analysis and homotopy for $M=\operatorname{Pr}=2$ and $k_{1}=-0.5$

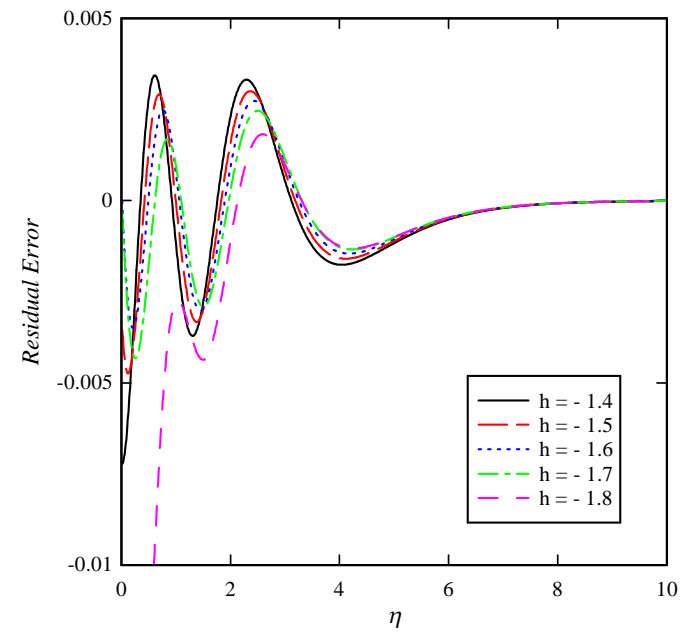

Fig. 3: Residual error equation (27) Twenty-stop solution for analysis and homotopy

$$
\text { . for } M=\operatorname{Pr}=2 \text { and } k_{1}=-0.5
$$

\section{Discussion and Results}

Nonlinear ordinary differential equations (8) and (9) have been resolved with respect to the proposed boundary conditions (10) and (11) in both fluid and second-degree fluid of Walters. Obviously, the visual representation can result in better understanding the impact of different parameters on the effective flow. The effect of various parameters has been investigated on curves, speed and temperature. 


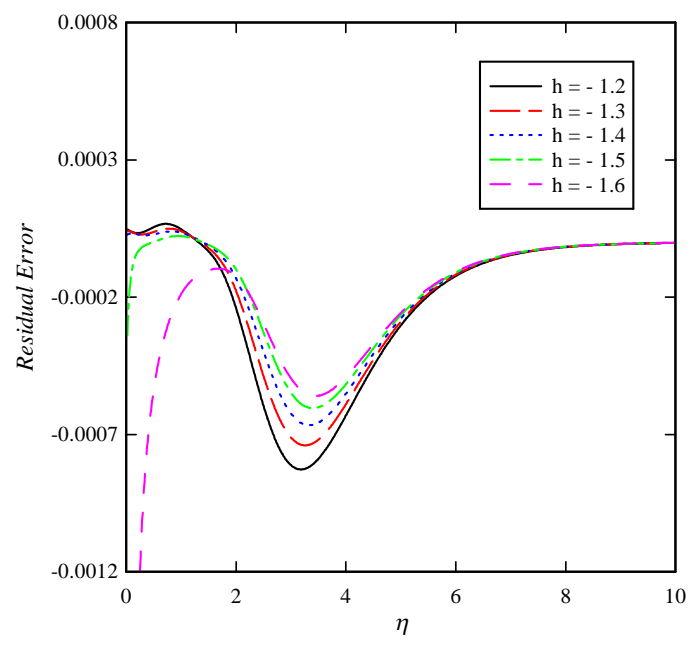

Fig. 4: The remaining error equation (28) Twenty-stop solution for analysis and homotopy

$$
\text { for } M=P r=2 \text { and } k_{1}=-0.5
$$

Viscoelastic parameters impact is shown on the velocity distribution shown in Figures 5 and 6. Viscoelastic tensile stresses and elastic deformation parameter of fluid particles are generated as a result of cross boundary layer contracts and thus decreases rapidly. However, this treatment is visible for Walters' fluid (second rank). It should be noted that, in reality, viscoelastic parameters are positive quantity based on laboratory results [14]. In addition, of course, if $k_{1}$ coefficient is equal to zero, second-order model becomes a model of Newtonian fluid. It is necessary to note that in some of the articles, other rheological models were used to model the fluid temperature only difference between these second rank fluids model is on $k_{1}$ mark. Due to the positive overall fluid properties and also due to the fact that the experimental results obtained on polymer solutions and melts are all showed positive results in these fluids are elastic so it can be argued that the results of fluid class non-value engineering and is considered merely a mathematical curiosity [15]. The entire thesis is intended both.

In this problem, viscoelastic parameter value is considered variable from $-0 / 5$ to $+0 / 5$. As it is clear fluid velocity from the unit in the wall reduced to zero in the free flow. While increasing $k_{1}$ in the fluid in Walters 'fluid decreases rapidly, and increases rapidly with increasing absolute value of viscoelastic parameters $\left|k_{1}\right|$.

Increasing the viscoelastic parameters for Walters' fluid due to reduce the amount of fluid increases the dimensionless temperature, while curve wall temperature gradient, while the opposite behavior is observed for secondary fluid [16]. Walters' fluid heat transfer rate decreases with increasing $k_{1}$. 
Figures 7 and 8, show magnetic parameters on velocity and temperature distribution curve. Such as drag force, called the Lorentz force arises due to the magnetic field applied perpendicular to the flow of electrical conductivity. This force tends to decrease with increasing temperature and flow rate near the screen. Therefore, the amount of speed reduction and thermal boundary layer thickness-increasing magnetic parameter increases. It should be noted that a large resistance on the fluid particles that generate heat in the fluid, caused by increased magnetic parameters.

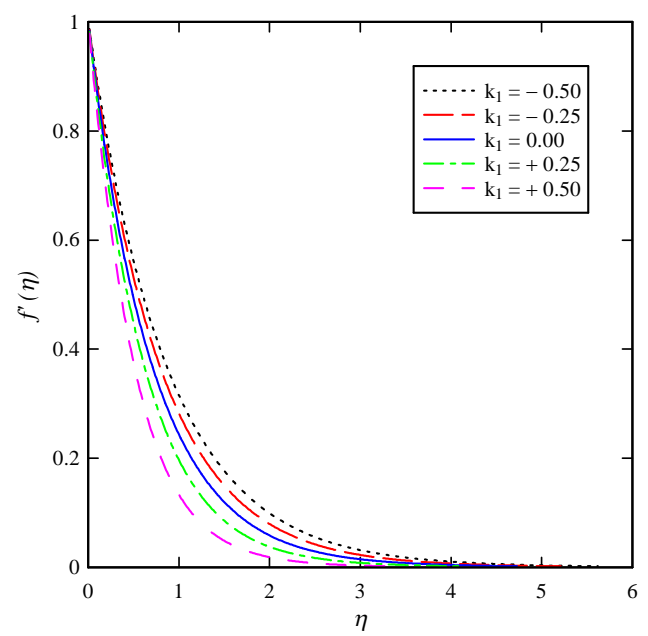

Fig. 5: Influence of viscoelastic parameters for speed curve for $M=\operatorname{Pr}=1$

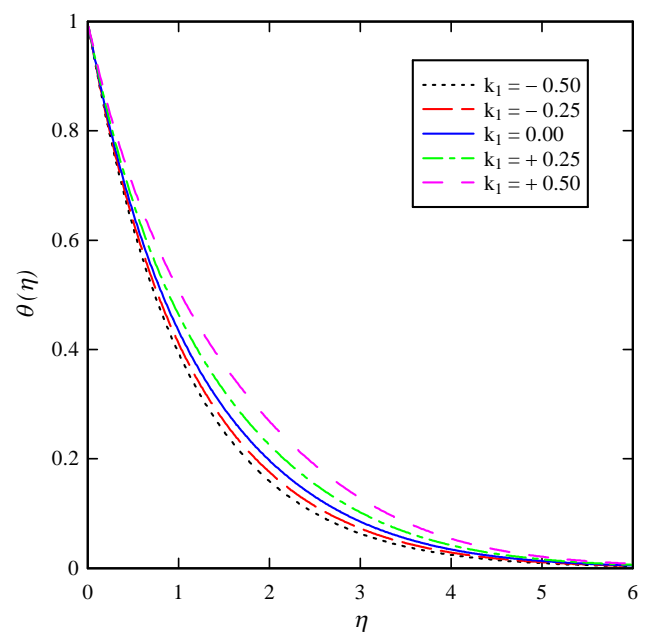

Fig. 6: The effect of temperature distribution curve for the viscoelastic parameters

$$
\text { for } M=\operatorname{Pr}=1
$$

The effect of Prandtl number on temperature distribution curve is shown in Figure 9. As important thermo-physical properties of the fluid, Prandtl number is defined as the ratio of heat to penetrate the middle momentum. For $\operatorname{Pr}<1$, thermal diffusivity of momentum precedes the momentum influence. The heat is released more quickly than momentum. In other words, small 
amounts of high conductivity and low viscosity fluids such as Prandtl number represents air and other gases. For $\operatorname{Pr}=1$ energy distribution rates and viscose like thermal boundary layer thickness and the speed (momentum) would be the same. For $\operatorname{Pr}>1$, the influence of thermal diffusivity overtakes momentum. In addition, the thermal conductivity of fluids such as water and oils few have the will and therefore with high levels of Prandtl number. Therefore, by increasing the number Prandtl, thermal boundary layer thickness decreases. On the other hand, it prevents the spread of heat flow with high Prandtl number fluid.

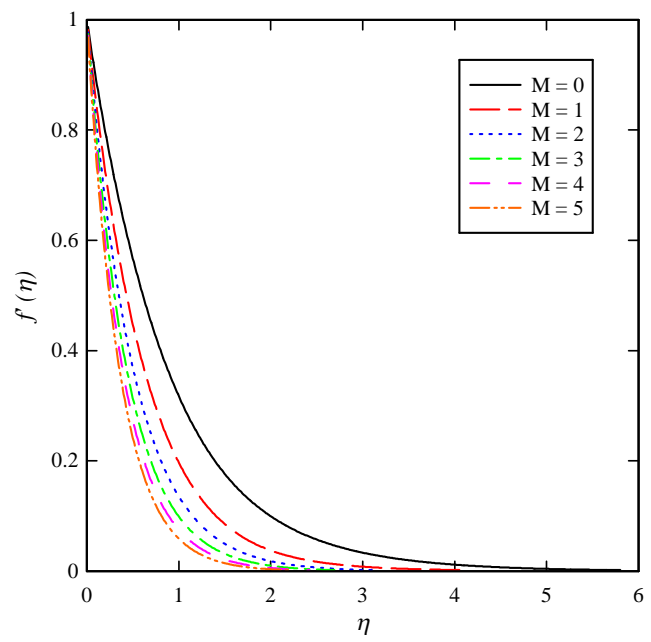

Fig. 7: Effect of magnetic parameters on speed for the curve for $k_{1}=0.25$ and $\operatorname{Pr}=1$

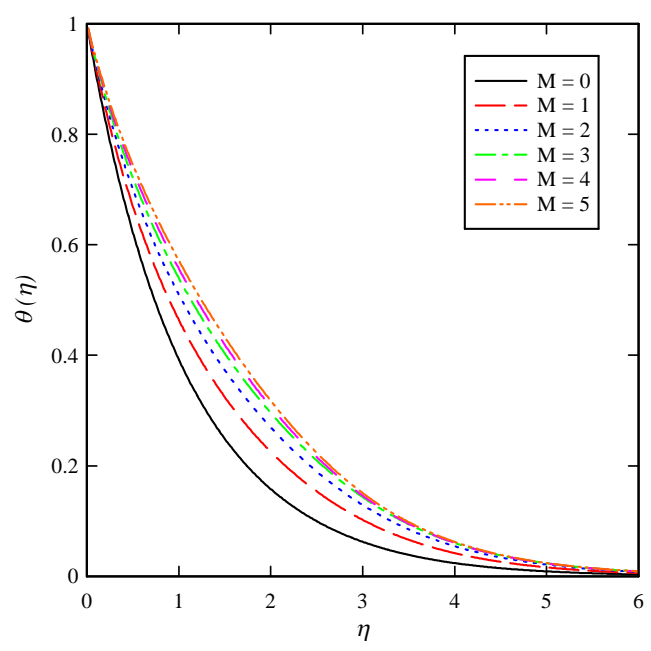

Fig. 8: Effect of magnetic parameters on temperature distribution curve for $k_{1}=0.25$ and $\operatorname{Pr}=1$ 


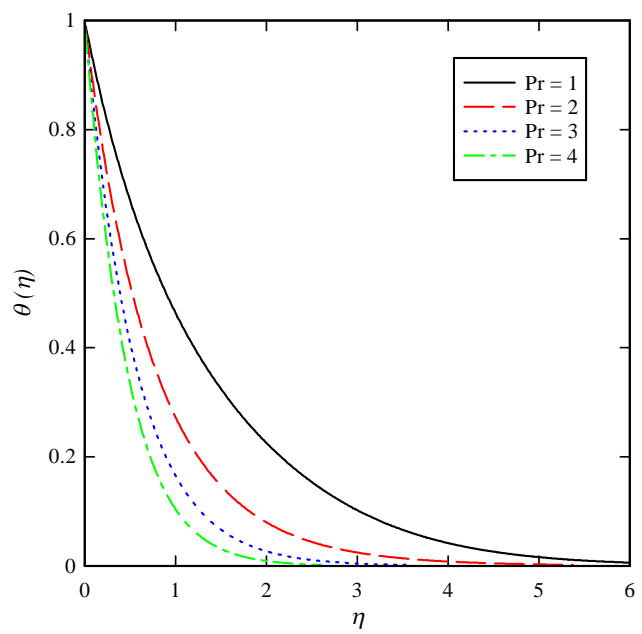

Fig. 9: Effect of Prandtl number on temperature distribution curve for $k_{1}=0.25$ and $M=1$

\section{Conclusion}

In this study, fluid flow and heat transfer in non-Newtonian viscoelastic fluid magnetic hydrodynamics steady laminar flow has been studied on a horizontal spreadsheet. Conservation equations are in the form of partial differential equations, ordinary differential equations, which, are used in order to convert them to a parallel solution for speed components as well as nondimensional temperature distribution. Dimensionless ordinary differential equations obtained are solved analytically using the homotopy analysis method. The results obtained in this study, other references and also used analytical solutions, which well be seen that shows the accuracy of the solution is in this article. Also, the influence of various physical parameters governing the viscoelastic parameter, magnetic parameter and Prandtl number was investigated on speed and temperature curves.

\section{References}

[1] K. Vajravelu, J. Nayfeh, Convective heat transfer at a stretching sheet, Acta Mechanica 96 (1993) 47-54.

[2] C.-K. Chen, M.-I. Char, Heat transfer of a continuous, stretching surface with suction or blowing, Journal of Mathematical Analysis and Applications 135 (1988) 568-580.

[3] R. G. Larson, Constitutive Equations for Polymer Melts and Solutions, Butterworths, 1988.

[4] A. Acrivos, M. J. Shah, E. E. Petersen, Momentum and heat transfer in laminar boundarylayer flows of non-Newtonian fluids past external surfaces, AIChE Journal 6 (1960) 312-317.

[5] W. R. Schowalter, The application of boundary-layer theory to power-law pseudoplastic fluids: Similar solutions, AIChE Journal 6 (1960) 24-28.

[6] J. H. Rao, D. R. Jeng, K. J. D. Witt, Momentum and heat transfer in a power-law fluid with arbitrary injection/suction at a moving wall, International Journal of Heat and Mass Transfer 42 (1999) 2837-2847. 
[7] R. S. R. Gorla, D. Voss, Heat transfer in a second order viscoelastic boundary layer flow at a stagnation point, International Journal of Engineering Science 18 (1980) 1301-1308.

[8] M. M. Nandeppanavar, K. Vajravelu, M. S. Abel, Heat transfer in MHD viscoelastic boundary layer flow over a stretching sheet with thermal radiation and non-uniform heat source/sink, Communications in Nonlinear Science and Numerical Simulation 16 (2011) 35783590.

[9] M. S. Abel, N. Mahesha, Heat transfer in MHD viscoelastic fluid flow over a stretching sheet with variable thermal conductivity, non-uniform heat source and radiation, Applied Mathematical Modelling 32 (2008) 1965-1983.

[10] K. V. Prasad, D. Pal, V. Umesh, N. S. P. Rao, The effect of variable viscosity on MHD viscoelastic fluid flow and heat transfer over a stretching sheet, Communications in Nonlinear Science and Numerical Simulation 15 (2010) 331-344.

[11] B. Siddappa, M. S. Abel, Non-Newtonian flow past a stretching surface, The Journal of Applied Mathematics and Physics 36 (1985) 890-892.

[12] S. J. Liao, On the homotopy analysis method for nonlinear problems, Applied Mathematics and Computation 147 (2004) 499-513.

[13] S. Liao, On the homotopy analysis method for nonlinear problems, Applied Mathematics and Computation 147 (2004) 499-513.

[14] R. B. Bird, R. C. Armstrong, O. Hassager, Dynamics of polymeric liquids, John Wiley \& Sons, 1987.

[15] K. Sadeghy, S. M. Taghavi, N. Khabazi, M. Mirzadeh, I. Karimfazli, On the use of hydrodynamic instability test as an efficient tool for evaluating viscoelastic fluid models, Advanced studies in theoretical physics 1 (2007) 367-379.

[16] C.-H. Chen, On the analytic solution of MHD flow and heat transfer for two types of viscoelastic fluid over a stretching sheet with energy dissipation, internal heat source and thermal radiation, International Journal of Heat and Mass Transfer 53 (2010) 4264-4273. 